\title{
Rhinoscopy assisted lacrimal probing - minimal invasive and effective therapeutic option for children with congenital nasolacrimal duct obstruction
}

MAGDALENA PRAUZIŃSKA ${ }^{A-F}$, BEATA PUCHER ${ }^{\mathrm{B}, \mathrm{D}, \mathrm{E}}$, AGATA KAŁUŻNA-MŁYNARCZYKK, ${ }^{\mathrm{B}}$, MICHAŁ KOTOWSKI ${ }^{B}$, JAGODA KOLASIŃSKA-LIPIŃSKA ${ }^{B}$, PAULINA MARIA ADAMCZYK ${ }^{B}$, JAROSŁAW SZYDŁOWSKIA, D

\author{
Pediatric Otolaryngology Department, Poznan University of Medical Sciences, Poland
}

A - Study Design, B - Data Collection, C - Statistical Analysis, D - Data Interpretation, E - Manuscript Preparation, F - Literature Search, G - Funds Collection

Summary Background. Congenital nasolacrimal duct obstruction (CNLDO) is a common developmental anomaly, and in most cases, obstruction is located at the distal end of the nasolacrimal duct. The rate of spontaneous resolution of CNLDO is up to $96 \%$ during the first year of life, and, therefore, most authors advise only medical treatment in the first months of life. When obstruction persists, lacrimal probing is performed. Conventionally, the next steps in surgical treatment are lacrimal intubation and dacryocystorhinostomy. The intranasal approach was first proposed many years ago, but became possible only after the development of advanced endoscopic rhinosurgery. At present, it is widely used to treat CNLDO.

Objectives. To evaluate the effectiveness of rhinoscopy assisted lacrimal probing as a treatment for congenital nasolacrimal duct obstruction in children.

Material and methods. We enrolled 14 children with CNLDO to this case series study. All children underwent surgery between 01.03.2016 and 30.03.2018. Data about symptoms and previous treatment were first collected, surgery was then performed, and all patients were then reexamined postoperatively and data about results recorded.

Results. The age range was 6-152 months (median 30 months). Four (4) patients had no history of previous surgery, and ten (10) had undergone lacrimal probing before. The overall final success rate was $85.71 \%$ (12/14 patients, including 2 infants with dacryocystitis). Conclusions. In our group of patients, endoscopy assisted lacrimal probing is an effective method of treatment for CNLDO. We suggest to use this method in cases of children older than 12 months with one or more unsuccessful probing, with infants with dacryocystitis, and always before considering dacryocystorhinostomy.

Key words: child, endoscopy, nasolacrimal duct.

Prauzińska M, Pucher B, Kałużna-Młynarczyk A, Kotowski M, Kolasińska-Lipińska J, Adamczyk PM, Szydłowski J. Rhinoscopy assisted lacrimal probing - minimal invasive and effective therapeutic option for children with congenital nasolacrimal duct obstruction. Fam Med Prim Care Rev 2018; 20(3): 255-258, doi: https://doi.org/10.5114/fmpcr.2018.78269.

\section{Background}

Congenital nasolacrimal duct obstruction (CNLDO) is a common developmental anomaly and a cause of visits in pediatric primary care. It results from a failure of canalization of the nasolacrimal duct, and affects up to $20 \%$ of normal newborns [1-4]. The diagnosis is made in a vast majority of cases (approximately $90 \%$ ) by a primary care physician, at a median age of 5 weeks without gender predilection. Premature birth has been shown be associated with the development of CNLDO. In most cases, it is caused by imperforated web at the level of Hasner's valve, which is located at the distal end of the nasolacrimal duct under the inferior nasal turbinate. Other reasons are narrowing of the inferior meatus, or, very uncommonly, bony obstruction of the nasolacrimal duct.

The nasolacrimal duct is not fully developed until the eighth month of gestation, and, therefore, infants born prematurely are less likely to have a fully patent duct $[1,4]$. The symptoms usually start shortly after birth when the natural increase of tear production takes place. The main symptom is usually epiphora, which is overflow of the tears with mattering of the lashes in one or both eyes. Subsequent overgrowth of bacteria in the obstructed nasolacrimal duct will result in constant/intermittent purulent or mucopurulent discharge, crusting on the lashes and recurrent conjunctivitis $[1,4]$. Besides an imperforated nasolacrimal duct, distally, there is also a valve-like obstruction proximally (at the junction of the common canaliculus and lacrimal sac), wherein a congenital dacryocystocele (congenital cyst of lacrimal sac) can develop. On occurrence, it is seen as a cystic mass of bluish coloration in the medial canthal region. In many cases, it might also have an intranasal extension. Due to the fact that infants are obligate nasal breathers, a bilateral nasolacrimal duct cyst can cause respiratory distress similar to that observed in children with bilateral choanal atresia. Dacryocystocele is prone to infection causing a form of infantile dacryocystitis [3].

The rate of spontaneous resolution of CNLDO is very high, up to $96 \%$ of all cases during the first year of life, and, therefore, most authors advise only medical treatment in first months of life $[1,5]$. This includes mainly hydrostatic massage of the lacrimal sac (Crigler massage) and ophthalmic antibiotic or antibiotic and steroid drops in cases of infection. Massaging the nasolacrimal sac in a downward direction creates hydrostatic pressure which can rupture the membranous obstruction. Antibiotic drops ameliorate inflammatory symptoms while waiting for resolution $[1,2,5]$.

When obstruction persists, lacrimal probing in local or general anesthesia is performed. Timing of this procedure re- 
mains controversial. While some authors strongly advise waiting 12 months to avoid unnecessary surgery, others advocate earlier (6-9 months) intervention to decrease the duration of symptoms $[1,5]$. The next step in surgical treatment according to ophthalmologist's therapeutic algorithms are lacrimal intubation and external dacryocystorhinostomy (DCR) $[1,5]$. Since the blockage site is located within the nose in a vast majority of cases, intranasal surgery to correct distal lacrimal obstruction was first proposed many years ago, but has become possible only after the development of advanced instruments and effective pediatric endoscopic rhinosurgery techniques. At the present time it is widely used to treat CNLDO $[3,6-8]$.

\section{Objectives}

To evaluate the effectiveness of rhinoscopy assisted lacrimal probing in treating congenital nasolacrimal duct obstruction in children.

\section{Material and methods}

\section{Study design}

The study was designed as a part of an interventional case series. Due to the non-experimental character of the study, a Bioethical Committee agreement was not required.

\section{Setting}

The study took place in the Pediatric Otolaryngology Department of Poznan University of Medical Sciences.

\section{Study size, participants and variables}

We enrolled 14 children with various symptoms of CNLDO. Data about symptoms, course of disease and previous treatment were collected. All children underwent surgery between 01.03.2016 and 30.03.2018, and it was performed under general anesthesia with intubation. At the beginning of the procedure, rhinosurgical gauze pledgets soaked on $0,1 \%$ adrenaline solution were placed in the nasal cavities. Thereafter, gentle nontraumatic probing of the inferior and superior lacrimal canaliculus and diagnostic syringing was performed. The pledgets were removed and endoscopy of the nasal cavities with use of a rigid 0 and/or 30 degree 2,7 mm endoscope was performed to reveal any existing nasal pathology. This was followed by medialization (infracture) of the inferior turbinate and the placing of a pledget under it on the affected side/s for a couple of minutes. After this, the inferior nasal meatus was visualized with endoscope and simultaneous syringing was performed to check patency of nasolacrimal duct. If no free flow of saline was observed, the mucosa of the lateral wall was perforated with a sickle knife or a ball probe. If patency of the lacrimal system was achieved in this way, the excessive mucosa from the distal end of the nasolacrimal duct was removed with a small straight or angled biting forceps. Flow of saline to the nose during irrigation indicated successful surgery. After surgery, antibiotic ophthalmic drops were prescribed for 14 days and parents/caregivers were told to perform hydrostatic massage of the lacrimal sac 3-5 times a day. All patients were reexamined postoperatively in the office facilities of our clinic and data about results were collected. If symptoms disappeared completely or nearly completely (ex. tearing only in wind or discharge only present during upper respiratory tract infection), it was judged as success.

\section{Results}

\section{Participants}

The study consisted of 14 children ( 8 boys and 6 girls) who underwent rhinoscopy assisted lacrimal probing and syringing. The age of the children ranged from 6 to 152 months, with a median of 30 months. The initial symptoms were: tearing and recurrent purulent discharge $(n=4)$, constant purulent discharge $(n=6)$, purulent discharge and swelling of eyelids $(n=2)$, and dacryocystitis $(n=2)$. Symptoms of 12 children at the time of surgery were unilateral (left -8 , right -4 ) and in two cases, bilateral. Of our patients, 4 had no history of previous surgery and 10 had undergone lacrimal probing before. In 4 of the latter, it was a single procedure and in 6, repeated (in 4-2 times, and 2-3 times).

\section{Main results}

In 12 children, surgery was successful (including all dacryocystitis cases), and in 2 unsuccessful (meaning not achieving patency of lacrimal system at the time of operation). During the examination that took place 1-3 months post-surgery, operation success was observed in 11/14 patients (78.57\%). In 9 of them, resolution of symptoms was complete, and in 2, near complete. In 2 children, there was no improvement, and in 1, only small improvement was observed. In one of girls with successful surgery and no improvement on a visit 6 weeks post operation, the parents had been found to be applying the wrong massage technique. After correction, the symptoms resolved completely in one month, which enabled the post-surgery success rate to reach $85.71 \%$. In all 7 children with successful surgery, a lack of symptoms was evident 1-3 months after surgery and as of observation time $>6$ months. Herein, good results were maintained over the whole observation time (7-24 months).

Figure 1 shows a 4 1/2 year-old boy with symptoms of CNLDO on the left side (mainly constant purulent discharge). Figure 2 shows the same boy one year later (4 months postoperation) with complete resolution of symptoms.

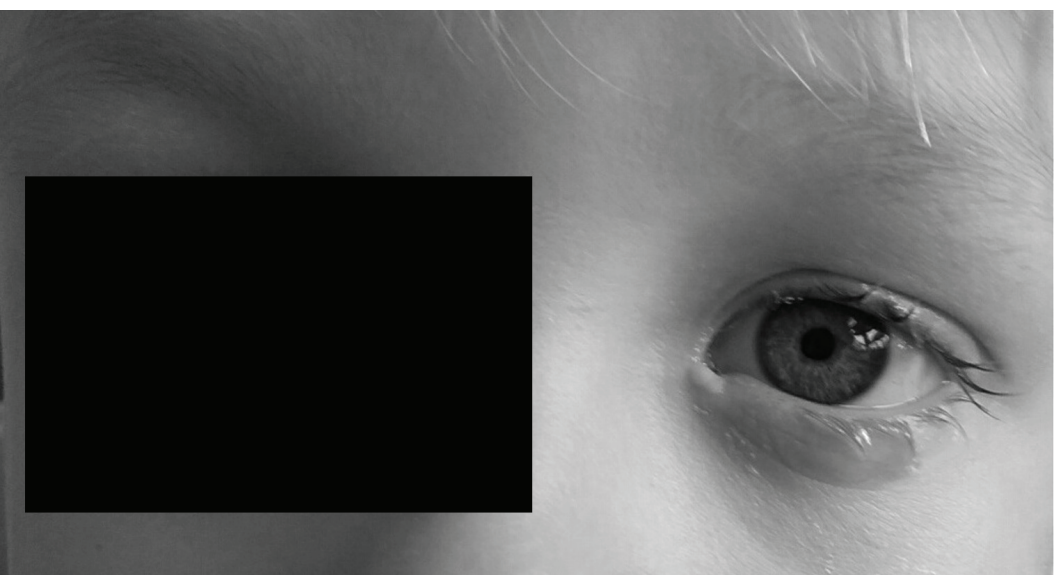

Figure 1. A 4,5 year boy with CNLDO on the left side after repeated $(3 \mathrm{x})$ unsuccessful probing 


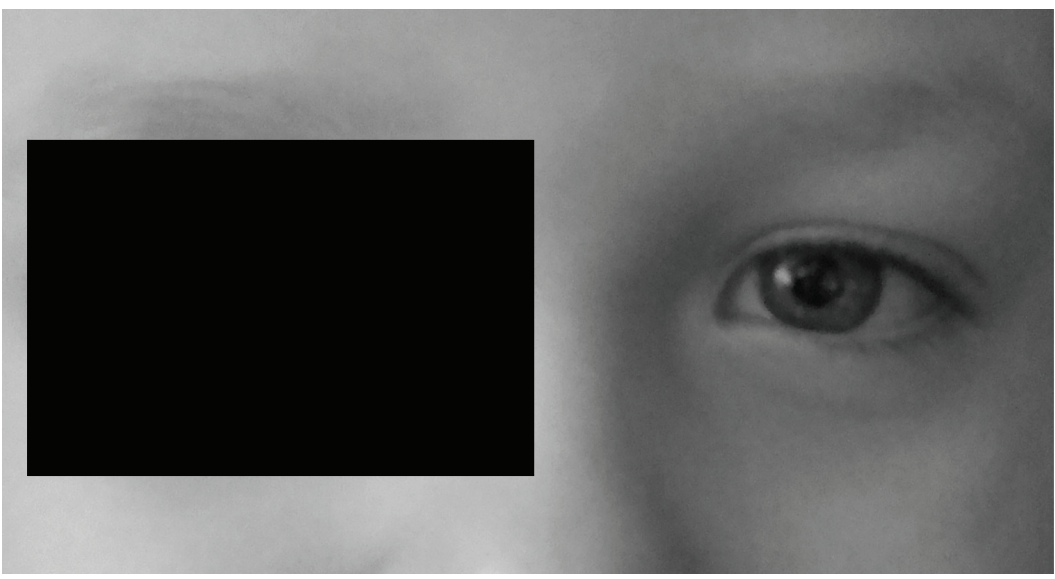

Figure 2. The same boy one year later 4 months after rhinoscopy assisted lacrimal probing

\section{Discussion}

Our group is relatively small, but our results $(85.71 \%$ success) are similar to that reported by other authors. Levin et al. [6] and Ali et al. [7], for example, used nasal endoscopy to treat congenital lacrimal sac mucoceles, including dacryocystitis, achieving, respectively, 23 cures in 24 infants [6] and a success rate of $86.6 \%$ [7]. In our work, we observed complete resolution of symptoms in 2 infants (age 6 and 8 months) with dacryocystitis. A second study also showed that good results are maintained over a long period of time (9 months) [7]. A further example is that of Wallace et al., who treated 67 children with all forms of CNLDO. For the entire group, probing resulted in a successful resolution of symptoms in $89 \%$. In opposition to our study, this one excluded patients who had had previous probings [8]. Moreover, Kouri et al., who used endoscopy assisted probing as a primary treatment in 40 children older than 12 months, saw an overall success rate $84.6 \%$ [9]. In addition, Galindo-Ferreiro et al., compared endoscopic assisted probing to conventional probing in children older than 48 months with $94.6 \%$ and $58.7 \%$ success rates in favor of endoscopic procedures. This study also included patients with a previous history of probing [10]. All the authors state that the use of an endoscope allows the identification of the site of obstruction and any coexisting intranasal anomaly. If one of these is a strongly lateralized, impacted, inferior turbinate, this alone can be a cause of CNLDO.

Conventional probing is a blind procedure, while endoscopy allows direct visualization of the distal end of the nasolacrimal duct. On of the most frequent reasons for unsuccessful probing is the so-called 'buried probe' or 'false probe passage'. This is a state wherein the probe does not perforate the mucosa of inferior meatus but remains in the submucosal layer of its lateral wall. In such cases, probing is unsuccessful despite a feeling of smooth passage even up to the floor of the nose. Conditions that may encourage false passage formation are anatomical variations of the nasolacrimal duct and thick nasal mucosa (which is typical for chronic inflammation). Gupta et al. deter- mined the prevalence of buried probe in complex CNLDO and founded it to come about in $8 \%$ of all cases [11]. During endoscopy, in contrast, the buried probe is clearly visible and possible to correct. A further benefit that increases the success rate of endoscopy is the possibility to create a wide opening instead of the small perforation that is made during blind probing.

These advantages make endoscopy assisted lacrimal probing much more effective than a conventional ('blind') probing $[3,8-11]$. Despite this, endoscopy assisted lacrimal probing is not a routine practice and is definitely much less commonly performed than conventional probing for many reasons. It requires general anesthesia and equipment such as pediatric endoscopic rhinosurgical instruments. What is more, the procedure has to be performed by a joint pediatric ENT/ophthalmology team or a surgeon who is familiar with both procedures.

After surgery, continuing massage of lacrimal sac is advised. This encourages a flow of tears through newly created opening. It is important, however, to make sure that parents/caregivers perform this in a proper (downward) fashion, because many are massaging in an upward direction, as it is easy to visibly empty the lacrimal sac this way. This happened with one of our patients, and we assume that it was a cause of lack of improvement on a first visit.

\section{Conclusions}

Endoscopy assisted lacrimal probing proved to be minimally invasive and effective in treating various types of congenital nasolacrimal duct obstruction in our group of patients. It has high success rates in children of all ages, whether as a primary treatment or after unsuccessful conventional probing. We suggest to use this method in cases of children older than 12 months with one or more unsuccessful probings, infants with dacryocystitis, and always before considering dacryocystorhinostomy. If symptoms like epiphora, recurrent conjunctivitis, constant purulent eye discharge, crusting in the line of eyelashes persist longer than 12 months or despite performed conventional probing, child should be referred for this type of treatment.

Source of funding: Paper developed using the university's funds (statute-based activity). Conflict of interest: The authors declare no conflict of interests.

\section{References}

1. Schnall BM. Pediatric nasolacrimal duct obstruction. Curr Opin Ophthalmol 2013; 24(5): 421-424.

2. Galindo-Ferreiro A, Palencia-Ercilla T, Mendoza Ferreira L, et al. A survey of management of congenital nasolacrimal duct obstruction by pediatric primary health care providers in Spain. Eur J Ophthalmol 2017; 27(4): 502-505.

3. Cunningham MJ. Endoscopic management of pediatric nasolacrimal anomalies. Otolaryngol Clin North Am 2006; 39(5): 1059-1074.

4. Sathiamoorthi S, Frank RD, Mohney BG. Incidence and clinical characteristics of congenital nasolacrimal duct obstruction. Br J Ophthalmol 2018; doi: 10.1136/bjophthalmol-2018-312074 [Epub ahead of print].

5. Heichel J, Bredehorn-Mayr T, Struck HG. Congenital nasolacrimal duct obstruction from an ophthalmologist's point of view: causes, diagnosis and staged therapeutic concept. HNO 2016; 64(6): 367-375. 
6. Levin AV, Wygnanski-Jaffe T, Forte $\mathrm{V}$, et al. Nasal endoscopy in the treatment of congenital lacrimal sac mucoceles. Int J Pediatr Otorhinolaryngol 2003; 67(3): 255-261.

7. Ali MJ, Singh S, Naik MN. Long-term outcomes of cruciate marsupialization of intra-nasal cysts in patients with congenital dacryocele. Int J Pediatr Otorhinolaryngol 2016; 86: 34-36.

8. Wallace EJ, Cox A, White P, et al. Endoscopic-assisted probing for congenital nasolacrimal duct obstruction. Eye (Lond) 2006; 20(9): 998-1003.

9. Kouri AS, Tsakanikos M, Linardos E, et al. Results of endoscopic assisted probing for congenital nasolacrimal duct obstruction in older children. Int J Pediatr Otorhinolaryngol 2008; 72(6): 891-896.

10. Galindo-Ferreiro A, Khandekar R, Akaishi PM, et al. Success rates of endoscopic-assisted probing compared to conventional probing in children 48 months or older. Semin Ophthalmol 2018; 33(3): 435-442.

11. Gupta N, Chawla N, Ganesh S, et al. Prevalence of buried probe in complex congenital nasolacrimal duct obstruction and evaluation of its success rate post 'probing and irrigation': a single-centre retrospective study. Orbit 2018; 5: 1-4.

Tables: 0

Figures: 2

References: 11

Received: 15.05 .2018

Reviewed: 24.05 .2018

Accepted: 21.07.2018

Address for correspondence:

Jarosław Szydłowski, MD, PhD

Klinika Otolaryngologii Dziecięcej UM

ul. Szpitalna 27/33

60-572 Poznań

Polska

Tel.: +48 61 849-13-63

E-mail: szydlowski@ump.edu.pl 